\title{
Perceived Orientation of Axis of Rotation in Structure-From-Motion
}

\author{
Corrado Caudek and Fulvio Domini \\ Cognitive Technology Laboratory, AREA Science Park
}

\begin{abstract}
Perceived orientation of axis of rotation and accuracy in discriminating fixed-axis from nonfixed-axis rotations were investigated for orthographic projections of three-dimensional rotating objects. The principal findings were (a) the slant of the axis of rotation was systematically misperceived; (b) in both two-view and multiview displays, the perceived slant of the axis of rotation was well-predicted by the ratio between the deformation (a property of the first-order optic flow) and the component parallel to the image plane of the global velocity vector; (c) if this ratio was kept constant in each frame transition of the stimulus sequence (or it was varied), then the stimuli tended to be judged as fixed-axis rotations (or as nonfixed-axis rotations), regardless of whether they simulated a fixed-axis rotation or not; and (d) the tilt of the axis of rotation was perceived in two-view displays with a very small error.
\end{abstract}

A changing two-dimensional (2-D) projection of an object's motion gives rise to a compelling impression of a volumetric shape moving in three-dimensional (3-D) space. This phenomenon (called the kinetic depth effect [KDE] after Wallach \& O'Connell, 1953) represents an essential task for the visual system and has received close psychophysical investigation. The research on the KDE has compared the properties of the projected objects recovered by the perceptual system with the properties recovered by a mathematical analysis of the stimulus information. Several psychophysical investigations have focused on the perceptual recovery of the structure of the projected objects. In particular, researchers have examined the discrimination between rigid and nonrigid motion (Braunstein, Hoffman, \& Pollick, 1990; Domini, Caudek, \& Proffitt, 1997; Norman \& Todd, 1993), the recovery of depth magnitudes (Braunstein, 1962; Braunstein \& Andersen, 1984; Caudek \& Proffitt, 1993; Domini \& Braunstein, in press; Durgin, Proffitt, Olson, \& Reinke, 1995; Eby, 1992; Liter, Braunstein, \& Hoffman, 1993; Loomis \& Eby, 1988, 1989; Proffitt, Rock, Hecht, \& Schubert, 1992; Todd, Akerstrom, Reichel, \& Haynes, 1988; Todd \& Bressan, 1990), and the perception of depth-order relations (Braunstein \& Andersen, 1981; Braunstein \& Tittle, 1988).

On the other hand, little attention has been given to the perceptual recovery of motion from structure-from-motion (SFM) displays. Kaiser (1990) investigated the perceptual recovery of angular velocities and found that discrimination thresholds for angular velocities of two simultaneously viewed solid cubes were similar to those for linear velocities

Corrado Caudek and Fulvio Domini, Cognitive Technology Laboratory, AREA Science Park, Trieste, Italy.

This research was supported in part by Consiglio Nazionale delle Ricerche Grant CT.94.00652.11. We thank J. Farley Norman, Frank E. Pollick, and an anonymous reviewer for helpful comments on earlier versions of this article.

Correspondence concerning this article should be addressed to Corrado Caudek, Cognitive Technology Laboratory, c/o INSIEL SpA, AREA Science Park, Padriciano 99, 34012 Trieste, Italy. Electronic mail may be sent to caudek@uts.univ.trieste.it.
(McKee, 1981). Kaiser and Calderone (1991) simulated rotating spheres defined by either random or regularly spaced texture elements on their surfaces. They also found that "the motion parameter accounting for most of the variance in observers' judgements is the true angular velocity, $\Omega$. Whereas extraneous spatiotemporal characteristics of the stimuli shift PSEs [points of subjective quality], these shifts are relatively minor" (p. 433). Even though these findings indicate good human sensitivity to the higher order parameters produced by distal angular velocities, it has been shown that the perception of angular velocities can be highly inaccurate. Domini et al. (1997) investigated the influence of a first-order property of the optic flow called deformation (def) on perceived angular velocities. In both the minimal conditions of three points and two views and in displays having a larger number of points and views, they found that perceived angular velocities were influenced by def, regardless of the simulated velocity, and that perceptual performance did not significantly improve if second-order temporal information was made available in the stimulus displays. The inconsistency between these two groups of results may depend on the fact that Kaiser and Kaiser and Calderone used (approximately) isotropic shapes, maintaining def roughly constant during rotation. The psychophysical evidence provided by Domini et al., therefore, suggests that angular velocities of rotating anisotropic shapes can be systematically misperceived (see also Pollick, 1994).

Another important aspect of perceived motion in SFM concerns the perceived orientation of the axis of rotation. Pollick, Nishida, Koike, and Kawato (1994) studied the ability to match finger orientation to the direction of the axis of rotation for full rotations of a group of discrete points, profiles of rotating ellipsoids, and two views of a group of discrete points. They found that observers were sensitive to both the simulated slant and the tilt of the axis of rotation but that sensitivity decreased as the axis of rotation approximated the viewing direction. Moreover, they found that observers provided consistent judgments about the orientation of the axis of rotation also when the stimulus displays did not provide information theoretically sufficient for 
uniquely specifying the slant of the axis of rotation (i.e., two-view displays). This finding has been interpreted by Pollick et al. (1994) as suggesting that observers introduce "an additional assumption to recover the axis of rotation" (p. 108). Consistent with this proposal, we hypothesized that perceived orientation of the axis of rotation in SFM is heuristically derived from the first-order properties of the optic flow. The present investigation is intended to extend our understanding of human perception of SFM by considering more closely the relation between the perceived orientation of the axis of rotation and the first-order optic flow.

\section{Properties of the Velocity Field Generated by a Moving Planar Patch}

In the present section, we review the mathematical properties of the first-order optic flow produced by the orthographic projection of a rotating planar patch, II. In particular, we consider the problem of recovering the orientation of the patch and the orientation of the axis of rotation.

Consider a Cartesian coordinate system $(x, y, z)$ centered at the observer. The orientation of a planar patch $I I$ in 3-D space can be described in terms of its slant $(\sigma)$ and tilt $(\tau)$. Slant is defined as the angle between the line of sight (i.e., the $z$-axis) and the normal to the patch. This angle varies over a range of $180^{\circ}$ and is equal to zero if the patch lies perpendicular to the line of sight (i.e., parallel to the $x-y$ plane). Tilt is defined as the angle between the projection into the $x-y$ plane of the normal to the patch and the $x$-axis.

If the patch $\Pi$ is undergoing rigid motion, then this motion can be decomposed into a translation and a rotation. The angular velocity vector, $\Omega$, in turn, can be decomposed into a component parallel to the $x-y$ plane $(\omega)$ and a component orthogonal to the $x-y$ plane ( $\rho$; see Figure 1). The angular velocity components about the $x, y$, and $z$ axes of the global angular velocity vector $\Omega$ are $\phi, \theta$, and $\rho . \phi$ and $\theta$ are the components of $\Omega$ in the $x-y$ plane.

Because orthographic projections have an inherent ambiguity that includes the axis of rotation (i.e., a clockwise

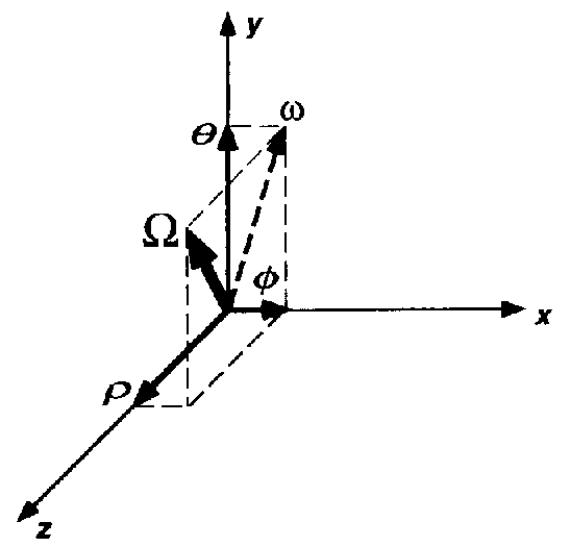

Figure 1. A generic velocity vector $\Omega$ can be decomposed into a component with modulus $\omega$ about an axis in the image plane (with components $\phi$ and $\theta$ in the directions $x$ and $y$ ) and into a component $\rho$ about an axis orthogonal to the picture plane. rotation of an axis with a slant of $60^{\circ}$ provides the same projected motion as a counterclockwise rotation with an axis of $120^{\circ}$ slant), we defined the slant of the axis of rotation as the minimum value of the two possible interpretations (i.e., the angle between the $z$-axis and the axis of rotation, or the angle of $180^{\circ}$ minus the angle between the $z$-axis and the axis of rotation). The tilt of the axis of rotation $\left(\tau_{\text {axis }}\right)$ was defined as the angle between the projection of the axis of rotation on the $x-y$ plane and the $x$-axis. The relation between $\sigma_{\text {axis }}$ and $\omega$ and $\rho$ is

$$
\sigma_{\mathrm{axis}}=\arctan \left(\frac{\omega}{\rho}\right)
$$

Hoffmann (1982) showed that the first-order properties of the velocity field are sufficient to determine (up to a reflection) (a) the tilt of the axis of rotation, $\tau_{\text {axis; }}$ (b) the component of the angular velocity $\Omega$ about the $z$-axis, $\rho$; and (c) the tilt of $\Pi, \tau$. Hoffman has also shown that the first-order properties of the velocity field are insufficient to uniquely determine (a) the slant of the axis of rotation, $\sigma_{\text {axis }}$, and (b) the slant of $\Pi, \sigma$.

From the first-order optic flow, however, it is possible to determine the product of $\omega$ and $\sigma$ (see Domini et al., 1997):

$$
\operatorname{def}=\sigma \omega
$$

This product is equal to one of the four components in which the optic flow is customarily decomposed. Koenderink and van Doorn $(1975,1976)$ showed, in fact, that the local instantaneous flow of a continuous velocity field can be uniquely decomposed into a translation, a rotation, a similarity (isotropic expansion), and a deformation (pure shape change or shear; see Figure 2). It should be noticed, however, that def does not uniquely specify the two parameters by which it is defined ( $\sigma$ and $\omega$ ). The first-order optic flow, in fact, is compatible with a one-parameter family of solutions for these parameters. This family of solutions can be represented by the loci of points of the hyperbola described by Equation 2 .

In conclusion, it is important to notice that the information provided by a two-view apparent motion sequence is not sufficient to define uniquely the slant of the axis of rotation $\left(\sigma_{\text {axis }}\right)$, the slant of the patch $\Pi(\sigma)$, and the modulus of the component in the $x-y$ plane of the global velocity vector $\Omega$ $(\omega)$. Nevertheless, also in these conditions, human observers provide consistent judgments about the slant of rotating planar surfaces (Domini, Caudek, \& Gerbino, 1995), the magnitude of angular rotation (Domini et al., 1997), and the slant of the axis of rotation (Pollick et al., 1994).

\section{Heuristic Recovery of the Slant of the Axis of Rotation from the Velocity Field}

Several investigators have suggested that human perception of SFM might depend primarily on the first-order optic flow. One source of evidence in this regard comes from the finding that perceptual performance improves very little, if 


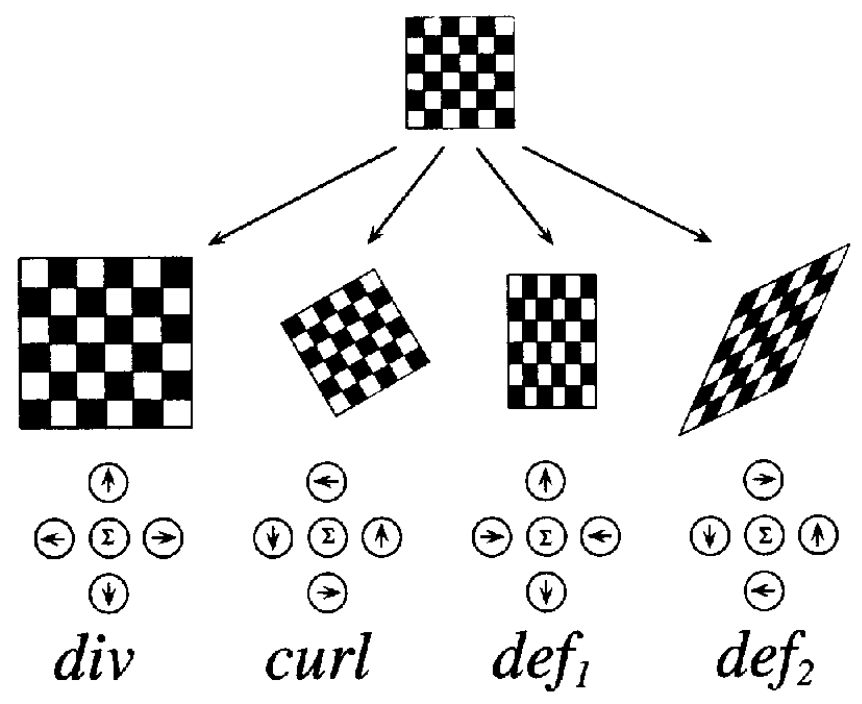

Figure 2. Geometrical transformations illustrating div (isotropic expansion), curl (rotation), $\operatorname{def}_{1}$, and def $_{2}$. Each of these components of the first-order optic flow can be specified by a scalar. The deformation (shear) is computed as: def $=\sqrt{\operatorname{def}_{1}^{2}+\operatorname{def}_{2}^{2}}$. def $=$ deformation. Adapted from "Optic Flow," by J. J. Koenderink, 1986, Vision Research, 26, p. 169. Copyright 1986 by Elsevier Science Ltd. Adapted with permission.

at all, if additional frames are added to a two-frame apparent motion sequence (Liter, Braunstein, \& Hoffman, 1993; Norman \& Todd, 1993; Todd \& Bressan, 1990; see also Eby, 1992; Hildreth, Grzywacz, Adelson, \& Inida, 1990; Pollick, 1997). Other evidence indicates that perceived SFM can be systematically biased by manipulating the first-order optic flow, even if second-order temporal information is made available in the stimulus displays (Caudek, Domini, \& Gerbino, 1994; Domini \& Braunstein, in press; Domini et al., 1995; Domini, Caudek, \& Proffitt, 1994; Domini et al., 1997). The present investigation intended to extend these previous findings by studying the relation between the first-order properties of the optic flow and the perceived orientation of the axis of rotation.

With the present experiments, we attempted to answer two related questions: (a) Is it possible to predict the slant of the axis of rotation perceived in two-view displays from the first-order properties of the optic flow? and (b) is it possible to predict the slant of the axis of rotation perceived in multiview displays from the first-order properties of the optic flow?

To answer these questions, we designed three experiments that were based on the hypothesis that the perceived slant of the axis of rotation $\left(\sigma_{a x i s}^{\prime}\right)$ is recovered through a heuristic analysis of the first-order optic flow. The proposed heuristic analysis was based on three assumptions:

1. $\omega$ is perceptually derived as a monotonic increasing function of def, $\omega^{\prime}=f_{\text {def }}$ (def). Evidence supporting this hypothesis has been provided by Domini et al. (1995) and by Domini et al. (1997).

2. $\rho^{\prime}$ is perceptually derived as a monotonic increasing function of the simulated parameter (because the first-order properties of the velocity field are sufficient to specify $\rho$ ), $\rho^{\prime}=f_{\rho}(\rho)$.

3. The global perceived velocity vector $\left(\Omega^{\prime}\right)$ is computed as the vector summation of the perceived components $\omega^{\prime}$ and $p^{\prime}$ (see Figure 1).

According to this heuristic analysis, then, $\sigma_{a x i s}^{\prime}$ is equal to

$$
\sigma_{\text {axis }}^{\prime}=\arctan \left(\frac{\omega^{\prime}}{\rho^{\prime}}\right)=\arctan \left(\frac{f_{\text {def }}(\text { def })}{f_{\mathrm{p}}(\rho)}\right) .
$$

If human perception of SFM conforms to this heuristic analysis, then we would expect that $\sigma_{\text {axis }}$ would be consistently judged correctly in two-view displays and systematically misperceived in multiview displays. Equation 3 can be applied to multiview displays by considering the average of the def magnitudes computed for each frame transition of the stimulus sequence. In particular, Equation 3 predicts a monotonic decrease of $\sigma_{a x i s}^{\prime}$ when $f_{\rho}(\rho)$ increases [and $f_{\text {def }}($ def $)$ is held constant] and a monotonic increase of $\sigma_{\mathrm{axis}}^{\prime}$ when $f_{\text {def }}($ def $)$ increases [and $f_{\mathrm{p}}(p)$ is held constant]. From this it follows that if the amount of the def variation is salient enough, $\sigma_{a x i s}^{\prime}$ would be perceived as varying even for simulated fixed-axis rotations. Conversely, if the ratio between def and $\rho$ remains approximately constant in each frame of the stimulus sequence, $\sigma_{\mathrm{axis}}^{\prime}$ would be perceived as unvarying even for simulated nonfixed-axis rotations.

\section{Experiment 1}

Experiment 1 tested the heuristic analysis described by Equation 3 by investigating the influence of def and $\rho$ on $\sigma_{\text {axis }}^{\prime}$. The stimuli provided only first-order temporal information (i.e., they were two-frame apparent motion sequences).

\section{Method}

Participants. Eight University of Trieste undergraduates participated in this experiment. All of them were naive to the purpose of the experiment.

Apparatus. The stimuli were presented on a high-resolution color monitor ( 1280 by 1024 addressable locations) under the control of a Silicon Graphics IRIS Workstation. The screen had a refresh rate of $60 \mathrm{~Hz}$ and was approximately photometrically linearized. An anti-aliasing procedure was used; for point-light locations falling on a pixel boundary, the screen luminance was proportionally adjusted in the relevant addressable locations. The graphics buffer was 8 bits deep ( 256 gray levels).

Participants viewed the displays through a reduction screen, which reduced the field of view to a circular area that subtended a visual angle of about $4^{\circ}$. The eye-to-screen distance was $1.1 \mathrm{~m}$.

Stimuli. The stimuli consisted of two views of 40 highluminance dots moving on a low-luminance background. The motions of the dots were computed as simulating an orthographic projection of planar surfaces undergoing oscillation in 3-D through an angular displacement of $6^{\circ}$. The stimulus onset asynchrony was equal to $215 \mathrm{~ms}$ with an interstimulus interval equal to zero. $\sigma_{\text {axis }}$ took on the values of $70^{\circ}, 50^{\circ}$, and $30^{\circ}$, and $\tau_{\text {aris }}$ was set to $90^{\circ}$. The axis of rotation was always contained in the simulated planar surfaces. In different stimulus displays, the initial slant of the simulated surfaces was varied by rotating the surfaces by $0^{\circ}, 20^{\circ}$, $40^{\circ}$, and $60^{\circ}$ from the position corresponding to the minimum slant 
Table 1

Stimulus Parameters for Experiment 1

\begin{tabular}{ccccc}
\hline $\begin{array}{c}\sigma_{\text {axis }} \\
(\mathrm{deg})\end{array}$ & $\begin{array}{c}\sigma \\
(\mathrm{deg})\end{array}$ & $\begin{array}{c}\gamma \\
(\mathrm{deg})\end{array}$ & $\begin{array}{c}\text { def } \\
(\mathrm{rad} / \mathrm{s})\end{array}$ & $\begin{array}{c}\rho \\
(\mathrm{deg})\end{array}$ \\
\hline 70 & 20.847 & 0 & .168 & 2.051 \\
70 & 32.371 & 20 & .260 & 2.051 \\
70 & 49.249 & 40 & .462 & 2.051 \\
70 & 67.529 & 60 & .881 & 2.051 \\
50 & 40.371 & 0 & .313 & 3.855 \\
50 & 46.487 & 20 & .367 & 3.855 \\
50 & 57.849 & 40 & .526 & 3.855 \\
50 & 71.846 & 60 & .913 & 3.855 \\
30 & 60.182 & 0 & .422 & 5.194 \\
30 & 63.294 & 20 & .460 & 5.194 \\
30 & 69.676 & 40 & .592 & 5.194 \\
30 & 78.266 & 60 & .949 & 5.194 \\
\hline
\end{tabular}

allowed by the orientation of the axis of rotation (i.e., the orientation of the surfaces with $\tau=90^{\circ}$ ). Because simulated angular velocity was kept constant, the initial slant of the planar surfaces affected the def of the stimulus displays. $\sigma_{\text {axis }}$, the initial slant of the simulated surfaces $(\sigma)$, the magnitude of rotation of the simulated surfaces from the minimum slant position $(\gamma)$, def, and $\rho$ for the different stimulus conditions are shown in Table 1.

The dots were randomly distributed with uniform probability density over the projection plane (not evenly distributed over the simulated surfaces). Dot lifetime was manipulated to keep the number of dots constant in each frame of the stimulus display. The dot density on the simulated surface was computed to project 40 dots within the unmasked portion of the stimulus displays in the first frame. In the successive frame, the number of visible dots was calculated. If there were $n$ dots in excess of 40 , then $n$ dots were randomly deleted from the stimulus displays. Given the small angle of rotation used in the present experiment, no more than $10 \%$ of the dots had only one frame lifetime.

An icon made up of two line segments forming an angle was shown in the right part of the terminal screen. One line segment represented the image plane as seen from the side and was oriented vertically. The other line segment represented the axis of rotation. Movement of a mouse connected to an IRIS Workstation varied the represented angular magnitudes in the range of $\pm 90^{\circ}$. The initial angular magnitude represented by the icon was randomly selected on each trial.

Design. Two independent variables were manipulated in this experiment: (a) the slant of the axis of rotation $\left(70^{\circ}, 50^{\circ}\right.$, and $\left.30^{\circ}\right)$ and (b) the initial slant of the simulated surfaces ( $\sigma$ was varied by rotating the surfaces by $0^{\circ}, 20^{\circ}, 40^{\circ}$, and $60^{\circ}$ from their minimum slant position). All variables were within-subjects. Each observer viewed 4 presentations in random order of the 12 combinations of the within-subjects variables. Twenty-four additional trials were presented at the beginning of each experimental session in order to familiarize the observers with the stimulus displays.

Procedure. All observers were run individually in one session. The observers were instructed to manipulate an icon present in the right part of the terminal screen in order to represent the perceived angle between the axis of rotation and the image plane. The perceived slant of the axis of rotation was then computed as the angle of $90^{\circ}$ minus the angle provided by the settings of the observers. Vision was monocular. Head motion was not restricted. Eye movements were permitted. While the experiment was run, the experimental room was dark. No restriction was placed on viewing time. No feedback was given until after the experiment was completed.

\section{Results and Discussion}

The perceived slant of the axis of rotation as a function of the initial orientation of the simulated planes for the three simulated slants of the axis of rotation is shown in Figure 3. A repeated-measures analysis of variance (ANOVA) indicated that $\sigma_{\text {axis }}^{\prime}$ was significantly affected by $\sigma_{\text {axis }}, F(2,6)=$ $33.748, p<.001, \eta^{2}=.92$. This finding is consistent with what Pollick et al. (1994) found. For two-views of seven points placed within the volume of spheres rotating at a constant angular velocity, Pollick et al. found that a simple linear model predicting $\sigma_{\mathrm{axis}}^{\prime}$ from $\sigma_{\mathrm{axis}}$ explained $83 \%$ of the variance for experienced observers and $61 \%$ of the variance for naive observers. In the present case, however, a regression model relating $\sigma_{\text {axis }}$ to the average $\sigma_{\text {axis }}^{\prime}$ for each cell of the experimental design $\left(\bar{\sigma}_{\text {axis }}\right)$ fitted the data very poorly, $\left.\bar{\sigma}_{\text {axis }}=62.747+.176 \sigma_{\text {axis }}, R^{2}=.06\right)$. According to our hypothesis, in fact, $\sigma_{\text {uxis }}^{\prime}$ depends on both $\rho$ (which covaries with $\sigma_{a x i s}$ in the present stimulus displays) and def. In this experiment, we manipulated def by varying the initial slant of the simulated surfaces and by keeping angular velocity constant. Consistent with our hypothesis, we found that the initial orientation of the simulated surfaces significantly affected $\sigma_{\text {axis }}^{\prime}, F(3,5)=228.133, p<.001, \eta^{2}=.99$. The interaction between the two independent variables was not significant.

One point to notice is that the heuristic analysis described by Equation 3 explains the significant correlation between perceived and simulated slant of the axis of rotation found by Pollick et al. (1994) in their two-view displays. If one keeps angular velocity constant, in fact, $\omega$ is a monotonic

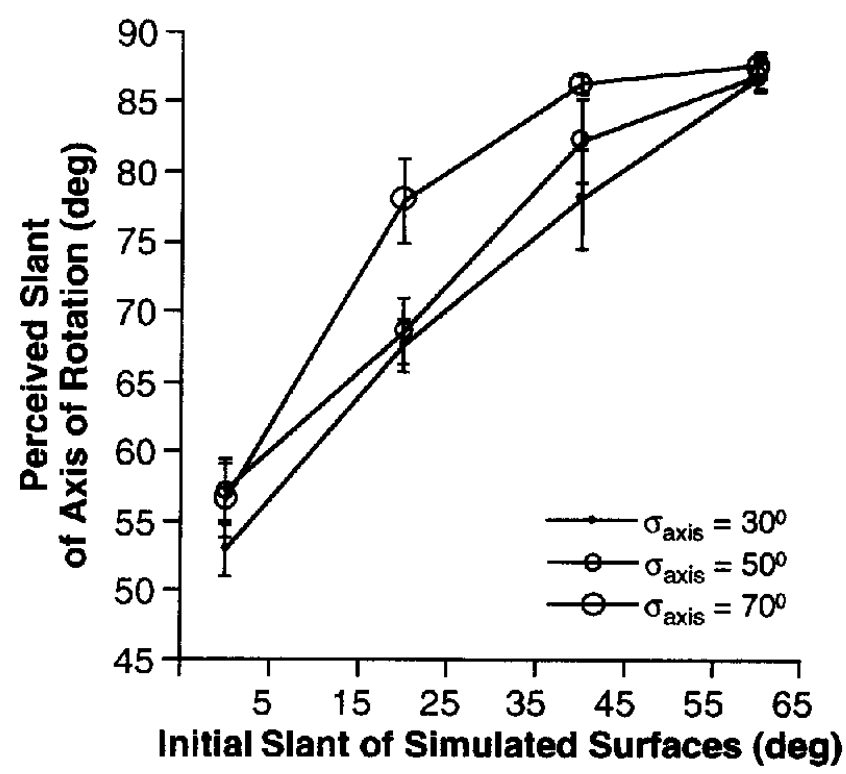

Figure 3. The average judged slant of the axis of rotation in Experiment 1 as a function of the initial orientation of the simulated surfaces for the three simulated slants of the axis of rotation. The initial slant of the simulated surfaces was varied by rotating the surfaces by $0^{\circ}, 20^{\circ}, 40^{\circ}$, and $60^{\circ}$ from their minimum slant position. Vertical bars represent one standard error. 
increasing function of $\sigma_{\text {axis }}$ and $\rho$ is a monotonic decreasing function of $\sigma_{\text {axis. }}$. Moreover, for points randomly distributed in a spherical volume, the mean def (defined as the product of $\omega$ and the average of the slant of each triplet of points, $\bar{\sigma}$ ) is a monotonic increasing function of $\sigma_{\text {axis }}$ (because $\bar{\sigma}$ is not influenced by the orientation of the sphere, $\overline{\boldsymbol{\sigma}}=\mathrm{k}$ ). From this it follows that for the displays used by Pollick et al., the magnitudes of $\sigma_{\text {axis }}^{\prime}$ estimated by Equation 3 are a monotonic increasing function of $\sigma_{\mathrm{axis}} \cdot{ }^{1}$ The heuristic analysis described by Equation 3, therefore, provides a valid model for the systematic judgments about the slant of the axis of rotation found by Pollick et al. in two-view displays.

Figure 4 shows the same data of Figure 3 plotted as a function of def and $\rho$. Consistent with the prediction of Equation 3, this figure shows that the magnitudes of $\sigma_{\text {axis }}^{\prime}$ decrease as $\rho$ increases (for constant def) and increase as def increases (for constant $p$ ).

A direct comparison of the predictions of Equation 3 with the observers' judgments would require knowledge of the functions $f_{\text {def }}$ and $f_{\mathrm{p}}$ relating the perceived and simulated parameters. Even though $f_{\text {def }}$ and $f_{\rho}$ are unknown, it is worthwhile to consider the hypothesis that they are simple unit operators, $f_{\text {def }}$ (def) $=$ def and $f_{\mathrm{p}}(\rho)=\rho$. A regression analysis performed with the introduction of this simplifying assumption indicated that $\bar{\sigma}_{\text {axis }}^{\prime}$ was well-predicted by the arc tangent of the ratio between def and $\rho, \bar{\sigma}_{\text {axis }}^{\prime}=-214.988+$ $3.533 \mathrm{def} / \rho, R^{2}=.88$. Because there was a clear nonlinearity between predicted and observed data, we applied a logarithmic transformation to the predictor, $\sigma_{\mathrm{axis}}^{*}=b_{0}+b_{1} \log \left(b_{2}\right.$ def $/ \rho+b_{3}$ ). In this way we obtained a further improvement of the fit, $\bar{\sigma}_{\mathrm{axis}}^{\prime}=11.07+.852 \sigma_{\mathrm{axis}}^{*}, R^{2}=.97$. The plot between observed and predicted data is shown in Figure 5 .

In conclusion, the results of Experiment 1 indicated that observers provided consistent judgments about the slant of the axis of rotation even if the stimulus information was not

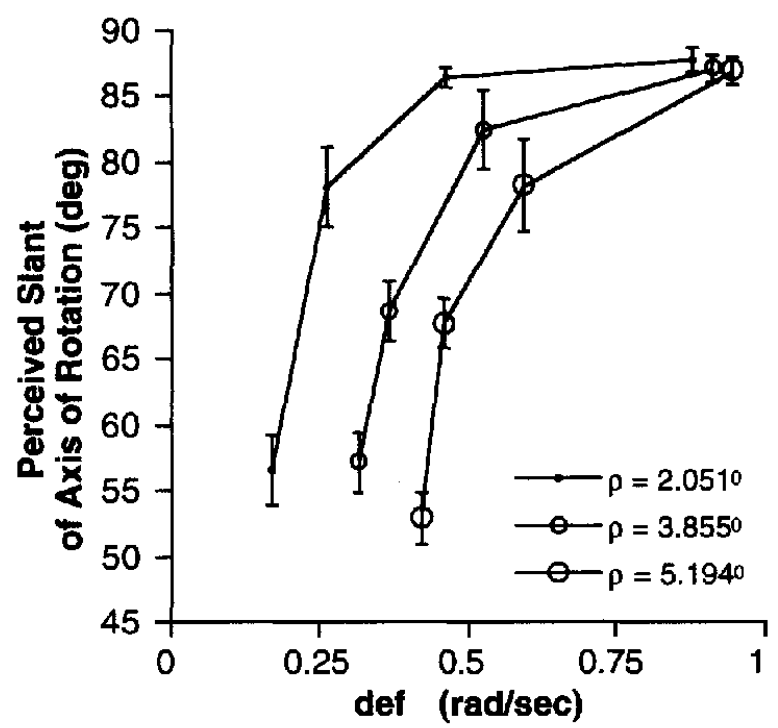

Figure 4. The average judged slant of the axis of rotation in Experiment 1 as a function of def for the three simulated $\rho$. Vertical bars represent one standard error. def $=$ deformation.

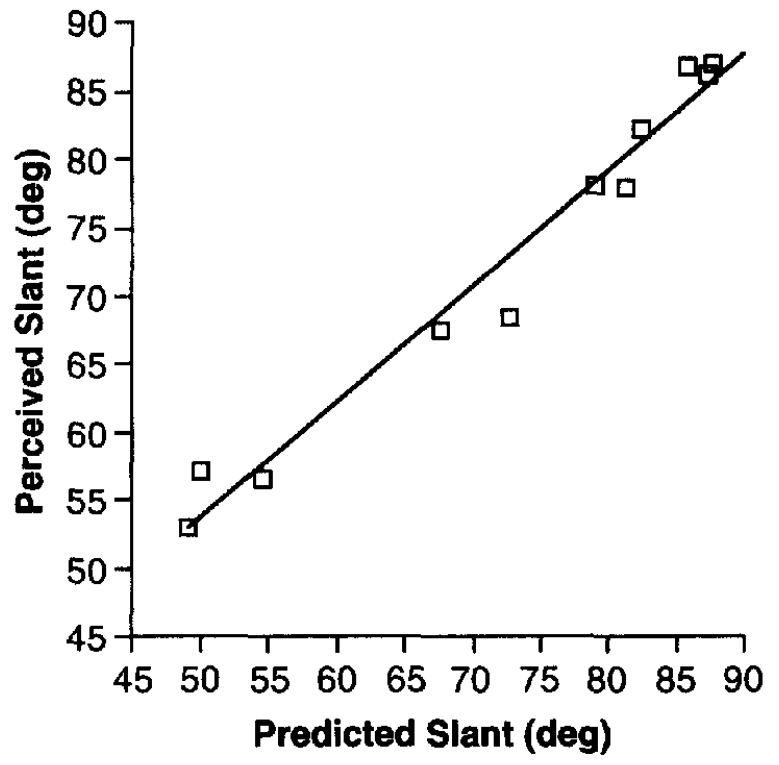

Figure 5. Mean judged slant of the axis of rotation versus predicted slant in Experiment 1.

sufficient to specify uniquely this property of the projected motion. Moreover, the perceived slant of the axis of rotation was accurately predicted from the properties of the firstorder optic flow according to Equation 3.

\section{Experiment 2}

In this experiment the perceived tilt of the axis of rotation was measured in two-frame apparent motion sequences. We expected performance to be accurate because two views are theoretically sufficient to derive this parameter of the simulated motion.

\section{Method}

Participants. Five observers participated in this experiment, including both of us and 3 University of Trieste undergraduates who were naive to the purpose of the experiment and had no previous experience with SFM displays.

Apparatus. The apparatus was the same as in Experiment 1.

Stimuli. The stimuli were the same as those of Experiment 1, except for the fact that the tilt of the simulated axis of rotation was randomly determined on each trial. An icon made up of two line segments forming an angle was shown in the right part of the terminal screen. One line segment represented the $x$-axis of the image plane and was oriented horizontally, and the other line segment represented the axis of rotation. Movement of a mouse connected to an IRIS Workstation varied the represented angular magnitudes. The initial angular magnitude represented by the icon was randomly selected on each trial.

Design. The design was the same as in Experiment 1.

\footnotetext{
${ }^{1} \sigma_{\text {axis }}^{\prime}=\arctan \left(f_{\text {def }}(\operatorname{def}) / f_{\rho}(\rho)\right)=\arctan \left(f_{\text {def }}(k \omega) / f_{\rho}(\rho)\right)=$ $\arctan \left(f_{\text {def }}\left(k f_{\text {incr }}\left[\sigma_{\text {axis }}\right]\right) / f_{\rho}\left(f_{\text {decr }}\left[\sigma_{\text {axis }}\right]\right)\right)$.
} 


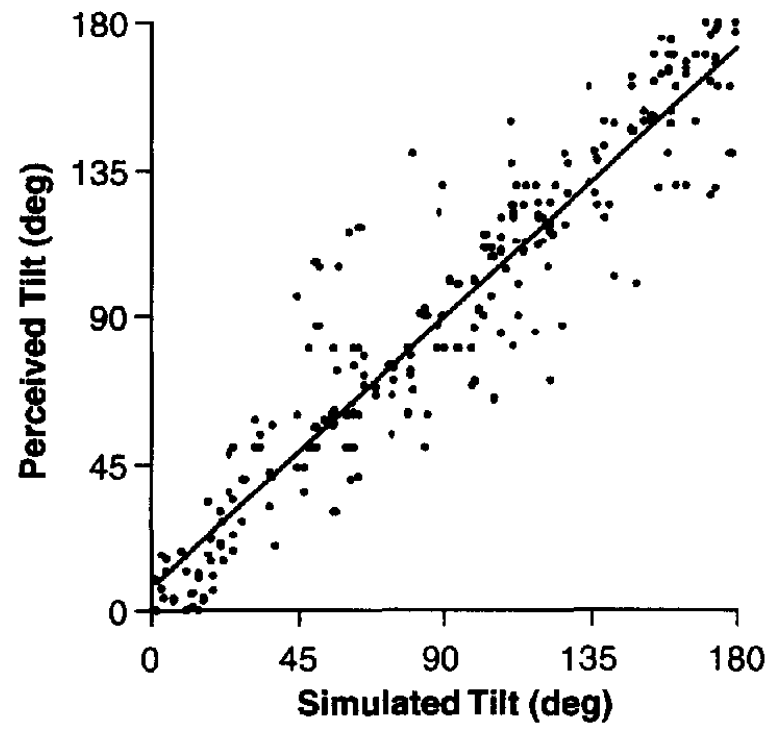

Figure 6. Scatterplot of perceived versus simulated tilt of the axis of rotation in Experiment 2.

Procedure. The observers were instructed to manipulate an icon present in the right part of the terminal screen in order to represent the perceived tilt of the axis of rotation. Otherwise, the procedure was the same as in Experiment 1.

\section{Results and Discussion}

A scatterplot of perceived versus simulated tilt of the axis of rotation is shown in Figure 6. A regression analysis indicated that the effect of simulated tilt $\left(\tau_{\mathrm{axis}}\right)$ on perceived tilt $\left(\tau_{\mathrm{axis}}^{\prime}\right)$ was significant, $t(236)=29.14, p<.001$, and the difference of the regression coefficients for expert and naive observers was not, $t(236)=-1.05, n s$. The overall proportion of the variance accounted for was equal to 0.88 . The magnitude of the (absolute) difference of the simulated and reported tilt magnitudes ranged from $8.54^{\circ}, S D=8.44$, for expert observers to $15.38^{\circ}, S D=14.54$, for naive observers, and this difference was statistically significant, $t(239)=$ $-4.164, p<.001$. These results indicate, therefore, that the data of the naive observers were noisier than those of the experts, but the results do not reveal any significant bias differentiating between the two groups. The regression coefficient representing the effect of simulated tilt on the reported tilt magnitudes was equal to 0.94 and differed significantly from 1 (veridical performance), $t(236)=$ $-2.732, p<.01$. The magnitude of this difference is small, though not negligible, and therefore indicates that both expert and naive observers can recover the tilt of the axis of rotation from two-view displays with a very small error.

\section{Experiment 3}

Experiment 3 investigated the effects of def and $p$ on $\sigma_{\text {axis }}^{\prime}$ in multiview displays. Different from the two-frame apparent motion sequences of Experiment 1, displays providing second-order temporal information allow in principle a veridical recovery of $\sigma_{\mathrm{axis}}$. For a generic fixed-axis rotation, in fact, the orthogonal projection of a point describes a segment of an elliptical path in the $x-y$ plane. The orientation and the eccentricity of this ellipse specify, respectively, $\tau_{\text {axis }}$ and $\sigma_{a x i s}$. Even if various SFM algorithms could be used for a veridical recovery of $\sigma_{\text {axis }}$ from these displays, we expected that the observers would not appropriately use the available second-order temporal information and would systematically misperceive $\sigma_{\text {axis }}$ according to Equation 3 .

\section{Method}

Participants. Eight University of Trieste undergraduates participated in this experiment. All of them were naive to the purpose of the experiment, and none of them participated in Experiments 1 and 2.

Apparatus. The apparatus was the same as in Experiment 1.

Stimuli. The stimuli were similar to those used in Experiment 1 and consisted of 60 views of 40 high-luminance dots moving on a low-luminance background. The motions of the dots simulated an orthographic projection of planar surfaces undergoing oscillation in 3-D through an angular rotation of $20^{\circ} . \sigma_{\text {axis, }}$, the initial slant of the simulated surfaces $(\sigma)$, the rotation of the simulated surfaces from the minimum slant position $(\gamma)$, mean def, the ratio between the standard deviation of the def magnitudes and mean def (SD/ $\operatorname{def}_{\text {mean }}$ ), and $\rho$ for the different stimulus conditions are shown in Table 2.

We used the same procedure as in the previous experiments to keep texture density constant. The dot density on the simulated surface was computed to project 40 dots within the unmasked portion of the stimulus displays in the first frame of the stimulus sequence. In each of the successive frames, the number of visible dots was computed. If there were $n$ dots in excess of 40 , then $n$ dots were randomly deleted from the stimulus displays.

Design and procedure. The design and the procedure were the same as in Experiment 1.

\section{Results and Discussion}

The perceived slant of the axis of rotation as a function of the initial orientation of the simulated planes for the three simulated slants of the axis of rotation is shown in Figure 7. A repeated-measures ANOVA indicated a significant effect of $\sigma_{\text {axis }}$ on $\sigma_{\text {axis }}^{\prime}, F(2,6)=13.425, p<.01, \eta^{2}=.82$.

Table 2

Stimulus Parameters for Experiment 3

\begin{tabular}{cccccc}
\hline $\begin{array}{c}\sigma_{\text {axis }} \\
(\mathrm{deg})\end{array}$ & $\begin{array}{c}\sigma \\
(\mathrm{deg})\end{array}$ & $\begin{array}{c}\gamma \\
(\mathrm{deg})\end{array}$ & $\begin{array}{c}\text { def } \\
(\mathrm{rad} / \mathrm{s})\end{array}$ & $\begin{array}{c}S D / \text { def }_{\text {mean }} \\
(\%)\end{array}$ & $\begin{array}{c}\rho \\
(\mathrm{deg})\end{array}$ \\
\hline 70 & 20.847 & 0 & .153 & 11.757 & 6.844 \\
70 & 32.371 & 20 & .26 & 16.997 & 6.844 \\
70 & 49.249 & 40 & .493 & 18.927 & 6.844 \\
70 & 67.529 & 60 & 1.153 & 33.192 & 6.844 \\
50 & 40.371 & 0 & .26 & 4.098 & 12.857 \\
50 & 46.487 & 20 & .34 & 10.305 & 12.857 \\
50 & 57.849 & 40 & .533 & 15.941 & 12.857 \\
50 & 71.846 & 60 & 1.18 & 32.098 & 12.857 \\
30 & 60.182 & 0 & .347 & 2.349 & 17.331 \\
30 & 63.294 & 20 & .407 & 7.141 & 17.331 \\
30 & 69.676 & 40 & .58 & 13.508 & 17.331 \\
30 & 78.266 & 60 & 1.2 & 30.963 & 17.331 \\
\hline
\end{tabular}




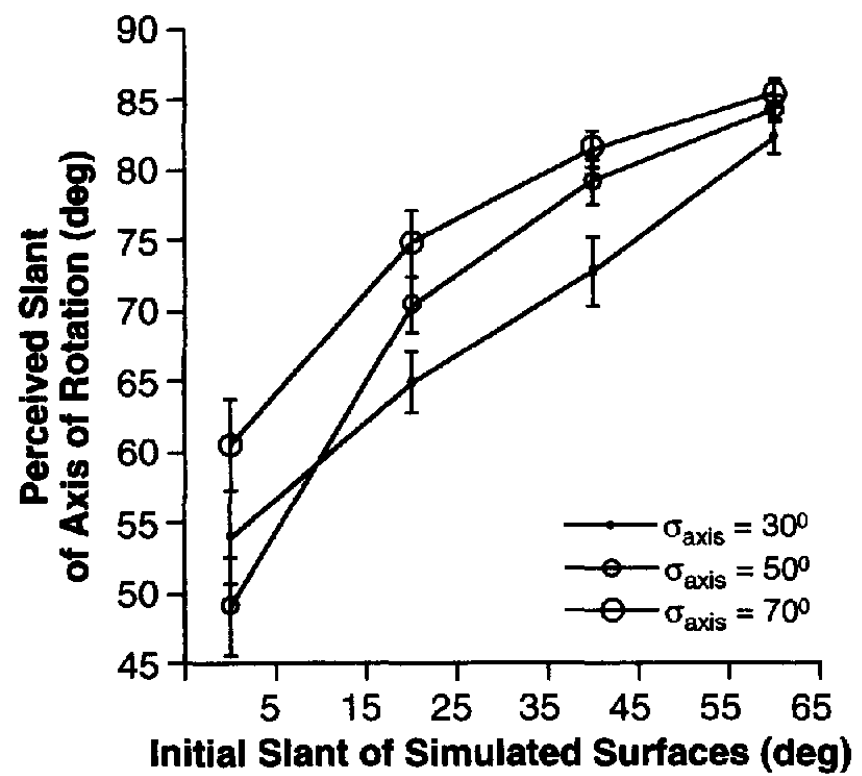

Figure 7. The average judged slant of the axis of rotation in Experiment 3 as a function of the initial orientation of the simulated surfaces for the three simulated slants of the axis of rotation. The initial slant of the simulated surfaces was varied by rotating the surfaces by $0^{\circ}, 20^{\circ}, 40^{\circ}$, and $60^{\circ}$ from their minimum slant position. Vertical bars represent one standard error.

However, as in Experiment 1, $\sigma_{\text {axis }}^{\prime}$ was also significantly affected by the initial orientation of the simulated surfaces, $F(3,5)=21.644, p<.01, \eta^{2}=.93$. The interaction between the two independent variables was not significant.

Figure 8 shows a plot of $\sigma_{a x i s}^{\prime}$ as function of def and $\rho$. From this figure we can see that even if the stimulus displays provided sufficient information for a veridical recovery of $\sigma_{a x i s}$, this aspect of the projected motion was systematically

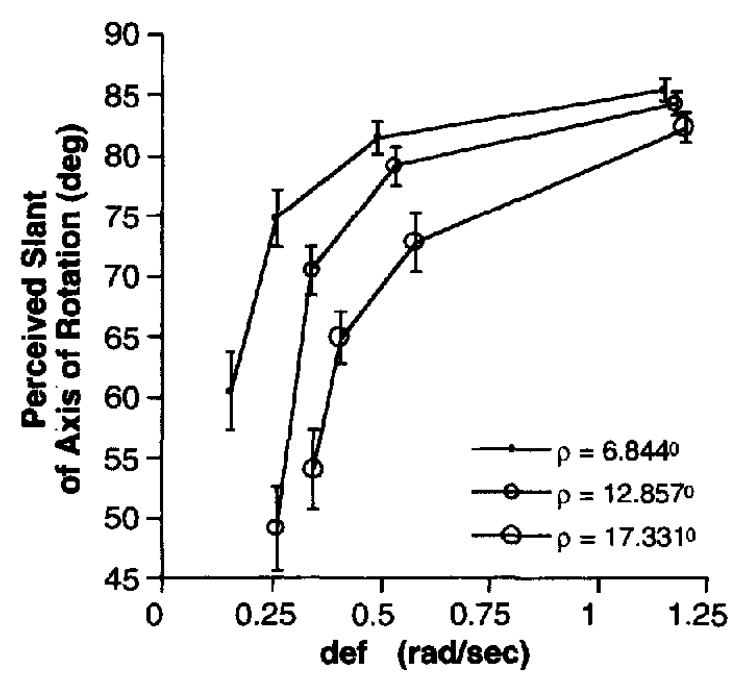

Figure 8. The average judged slant of the axis of rotation in Experiment 3 as a function of def for the three simulated $\rho$. Vertical bars represent one standard error. def $=$ deformation misperceived, in keeping with Equation 3. In fact, the magnitudes of $\sigma_{\text {axis }}^{\prime}$ decreased as $\rho$ increased (for constant def) and increased as def increased (for constant $\rho$ ). It should be noted that when $\rho$ was kept constant, $\sigma_{\text {axis }}$ was also constant.

A simple-regression analysis revealed that $\bar{\sigma}_{\text {axis }}^{\prime}$ was poorly predicted by $\sigma_{\text {axis }}, \bar{\sigma}_{\text {axis }}^{\prime}=62.747+.176 \sigma_{\text {axis }}, R^{2}=$ .06. Conversely, a regression analysis relating $\bar{\sigma}_{\text {axis }}^{\prime}$ to the arc tangent of the ratio between def and $\rho$ accounted for a large proportion of the variance, $\bar{\sigma}_{\text {axis }}^{\prime}=-134.579+2.533$ arc tangent $(\operatorname{def} / \rho), R^{2}=.91$. After applying a logarithmic transformation to the predictor, $\sigma_{\text {axis }}^{*}=b_{0}+b_{1} \log \left(b_{2}\right.$ $\operatorname{def} / \rho+b_{3}$ ), we obtained a further improvement of the fit, $\bar{\sigma}_{\text {axis }}^{\prime}=4.1+.952 \sigma_{\text {axis }}^{*}, R^{2}=.98$. The plot between observed and predicted data is shown in Figure 9.

The results of Experiment 3 are not in agreement with those of Pollick et al. (1994) because Pollick et al. found that $\sigma_{\text {axis }}$ was accurately perceived in multiview displays. For 200 views of 21 points randomly positioned within the volume of spheres continuously rotating by $360^{\circ}$, Pollick et al. found that a simple-regression model predicting $\sigma_{\mathrm{axis}}^{\prime}$ from $\sigma_{\text {axis }}$ fit the data very well (the variance accounted for was equal to $78 \%$ for experienced observers and to $55 \%$ for naive observers). The data of Pollick et al., however, are only apparently inconsistent with the present data because for isotropic shapes rotating at a constant angular velocity, Equation 3 makes it possible to recover from the first-order velocity field magnitudes of $\sigma_{\text {axis }}^{\prime}$ that are a monotonic increasing function of the simulated parameters (see the Results and Discussion section of Experiment 1). By means of a heuristic analysis of the first-order optic flow, therefore, it is possible to explain both the veridical performance found by Pollick et al. and the biases that limited performance in the present experiment.

In conclusion, three aspects of the findings of Experiment 3 deserve to be highlighted. First, $\sigma_{\text {axis }}$ was systematically

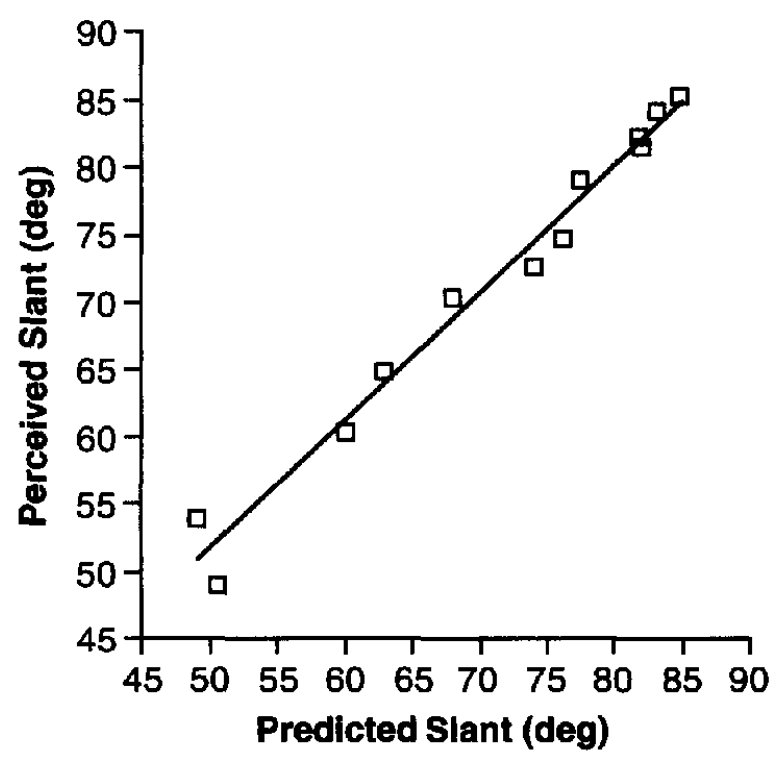

Figure 9. Mean judged slant of the axis of rotation versus predicted slant in Experiment 3. 
misperceived in multiview displays that simulated the rotation of planar surfaces. Second, the misperceptions found in Experiment 3 closely resemble the biases revealed by Experiment 1 . Third, the misperceptions of $\sigma_{\text {axis }}$ were well-predicted by the heuristic analysis of the first-order optic flow described by Equation 3.

\section{Control Experiment}

In Experiments 1-3, dot lifetime was manipulated in order to keep dot density constant. In this way, dot lifetime varied with the initial slant of the simulated surfaces. The percentage of dots with a lifetime smaller than the number of frames of the stimulus sequence increased with the initial slant of the simulated surfaces (this percentage was equal to about $10 \%$ for the smallest $\sigma_{a x i s}$ and the smallest initial slant of the simulated surfaces and about $55 \%$ for the largest $\sigma_{\text {axis }}$ and the largest initial slant of the surfaces). Even though scintillation (dot noncorrespondence) provides only a weak cue for 3-D shape perception (see Lappin, Doner, \& Kottas, 1980; Sperling, Landy, Dosher, \& Perkins, 1989), our experimental manipulation created a condition-dependent noise, possibly providing an extraneous cue. In order to control for this possible confound, in this control experiment dot density was not controlled, and therefore, the displays provided changing texture density cues consistent with the motions of the projected surfaces.

Five observers participated in this experiment, including both of us and 3 University of Trieste undergraduates who were naive to the purpose of the experiment. None of the observers participated in Experiments 1 and 3.

The stimuli were similar to those used in Experiment 3 and consisted of 60 views of 40 high-luminance dots moving on a low-luminance background. The motions of the dots simulated an orthographic projection of planar surfaces undergoing oscillation in 3-D through an angular rotation of $20^{\circ}$. The design and the procedure were the same as in Experiment 3. A 2 (expert vs. naive observers) $\times 3$ (simulated slant of axes of rotation) $\times 4$ (initial slant of simulated surfaces) repeated-measures ANOVA replicated the results of Experiments 1 and 3. The effect of $\sigma_{\text {axis }}$ on $\sigma_{\text {axis }}^{\prime}$ was significant, $F(2,6)=23.34, p<.01$; with the increase of $\sigma_{\text {axis }}$, the reported magnitudes of $\sigma_{\text {axis }}^{\prime}$ increased by $49 \%$. More important, the effect of the initial orientation of the simulated surfaces on $\sigma_{\text {axis }}^{\prime}$ was also significant, $F(3,5)=$ $18.8, p<.001$; with the increase of the initial orientation of the simulated surfaces, $\sigma_{\text {axis }}^{\prime}$ increased by $64 \%$. The effect of expertise was not significant, $F(1,3)=1.25$, ns. None of the interactions was significant.

As for Experiment 3, a simple-regression analysis revealed that $\bar{\sigma}_{\text {axis }}^{\prime}$ was poorly predicted by $\sigma_{\text {axis }}, \bar{\sigma}_{\text {axis }}^{\prime}=$ $63.945+.204 \sigma_{\text {axis }}, R^{2}=.17$. On the other hand, after applying a logarithmic transformation to the predictor, a regression analysis relating $\bar{\sigma}_{\text {axis }}^{\prime}$ to the arc tangent of the ratio between def and $\rho, \sigma_{\text {axis }}^{*}=b_{0}+b_{1} \log \left(b_{2} \operatorname{def} / \rho+b_{3}\right)$, accounted for a large proportion of the variance, $\bar{\sigma}_{\text {axis }}^{\prime}=$ $.015+.999 \sigma_{\mathrm{axis}}^{*}, R^{2}=.8$.

In conclusion, the results of the control experiment replicated those of Experiment 3, thus showing that the misperceptions revealed by the previous experiment cannot be accounted for by the scintillation of the stimulus displays.

\section{Experiment 4}

In the previous experiments, we found that the slant of the axis of rotation of oscillating planar surfaces was misperceived and that, in large part, these misperceptions were accounted for by the heuristic analysis described in Equation 3. Equation 3 is based on first-order information (i.e., the information provided by two views), and, in those experiments, the first-order properties of the stimulus displays did not vary (Experiments 1 and 2) or varied by a small amount (Experiment 3). In the present experiment, conversely, we created stimulus displays where the first-order properties varied largely over views so that we could test the sensitivity of the observers to the variations of def and $\rho$.

Observers were asked to discriminate fixed-axis rotations from nonfixed-axis rotations. By manipulating $\rho$ and def, we created four classes of displays: (a) nonfixed-axis rotations for which $\sigma_{\text {axis }}^{\prime}$ (computed in each frame transition according to Equation 3) remained nearly constant over the whole stimulus sequence, (b) fixed-axis rotations for which $\sigma_{\text {axis }}^{\prime}$ varied in the course of the stimulus sequence, (c) fixed-axis rotations for which $\sigma_{\mathrm{axis}}^{\prime}$ remained nearly constant over the whole stimulus sequence, and (d) nonfixed-axis rotations for which $\sigma_{a x i s}^{\prime}$ varied in the course of the stimulus sequence. If observers are sensitive to the variation of def and $\rho$ over time according to Equation 3, then we would expect that the third and fourth class of displays would be perceived veridically, whereas the first and second class of displays would be systematically misperceived.

\section{Method}

Participants. Sixteen University of Trieste undergraduates participated in this experiment. All of them were naive to the purpose of the experiment.

Apparatus. The apparatus was the same as in Experiment 1.

Stimuli. The stimuli consisted of 60 views of 40 highluminance dots moving on a low-luminance background. The motions of the dots simulated an orthographic projection of points placed either on planar surfaces (anisotropic shapes) or within spherical volumes (isotropic shapes) and oscillating in 3-D through an angular rotation of $70^{\circ}$. The planar surfaces were oriented so that the axis of rotation was contained in the simulated surfaces during the whole oscillation. The tilt of the surfaces was equated to the tilt of the axis of rotation at the beginning of each oscillation cycle. The spheres were simulated with the axis of rotation passing through their center. For both planar surfaces and spheres, the tilt of the axis of rotation was determined at random on each trial. Because different triplets of dots placed within rotating spherical volumes produce different def magnitudes, for the isotropic shapes def was computed as the mean of the deformations of each triplet of dots in each frame transition.

For fixed-axis rotations (FA condition), $\sigma_{\text {axis }}$ was equal to $60^{\circ}$. For nonfixed-axis rotations (NFA condition), there were two conditions. In the first condition, $\sigma_{\text {axis }}$ was changed from $90^{\circ}$ to $60^{\circ}$ in the first half-cycle of the simulated oscillations of dots and from $60^{\circ}$ to $90^{\circ}$ in the second half-cycle of the oscillations. Because for planar surfaces the magnitudes of $\rho$ and def varied in the same 
direction in successive frame transitions of the stimulus sequence (they both monotonically increased or monotonically decreased), this condition was called NFA in-phase condition (see Figures 10 and 11 ). In the second condition, $\sigma_{\text {axis }}$ was changed from $60^{\circ}$ to $90^{\circ}$ in the first half-cycle of the simulated oscillations of dots and from $90^{\circ}$ to $60^{\circ}$ in the second half-cycle of the oscillations. Because for planar surfaces the magnitudes of $\rho$ and def varied in opposite directions in successive frame transitions of the stimulus sequence (one increased and the other decreased), this condition was called NFA counterphase condition (see Figures 10 and 11). For displays simulating nonfixed-axis rotations of dots placed within spherical volumes, the in-phase and counterphase conditions were equivalent. In both cases, in fact, the magnitude of mean def remained approximately constant in successive frame transitions of the stimulus sequence (see Figure 10). Mean def was computed as the mean of the deformations produced by every triplet of points.

The dots were randomly distributed with uniform probability density over the projection plane (not evenly distributed over the simulated surfaces or within the simulated volumes). Dot lifetime was manipulated to keep the number of dots constant in each frame of the stimulus display. Each stimulus display was contained within a circular window with a diameter of about $4^{\circ}$ of visual angle.

A model consisting of a white cardboard surface with black dots painted on it was used to instruct the observers. The cardboard surface could rotate about a coplanar axis, and the orientation of the axis of rotation could be varied.

Design. Two independent variables were studied in this experiment: (a) shape (isotropic vs. anisotropic) and (b) axis orientation (FA, NFA in-phase, NFA counterphase). The variable shape was betweensubjects, and the variable axis orientation was within-subjects. Each observer viewed 80 displays in random order for each of the three levels of the within-subjects variable. Twenty-four additional trials were presented at the beginning of each experimental session in order to familiarize the observers with the stimulus displays.

Procedure. Each observer participated in one experimental session. Each session began with 24 practice trials (six repetitions of the three within-subjects conditions), followed by a sequence of 240 trials that consisted of 80 signal trials and 160 noise trials ( 80 trials for each of the NFA in-phase and NFA-counter-phase

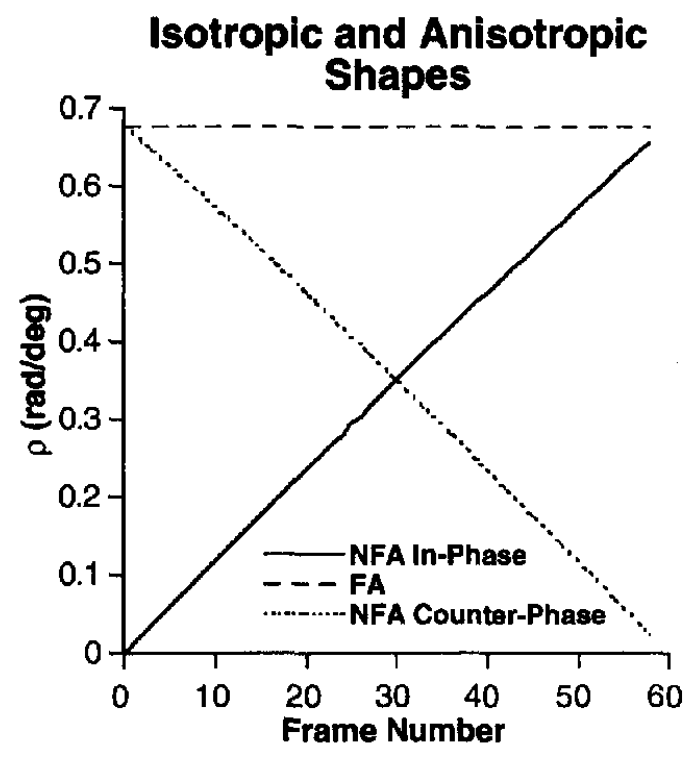

Figure 11. The rotatory velocity component about an axis parallel to the image plane ( $\rho$ ) as a function of frame transition in Experiment 4 for anisotropic and isotropic shapes in the NFA in-phase, FA, and NFA counterphase conditions. NFA = nonfixedaxis rotations; $F A=$ fixed-axis rotations.

conditions). The trials were completely randomized. The observers were allowed to rest in between trials.

The observers were instructed to press the "fixed-axis rotation" button if the simulated dots appeared to rotate about an axis steadily oriented in 3-D space and the "nonfixed-axis rotation" button otherwise. A cardboard model was used to demonstrate both the fixed-axis and the nonfixed axis conditions. The observers were told to perform as accurately as possible, without restrictions on viewing time. They were informed that fixed-axis rotations would

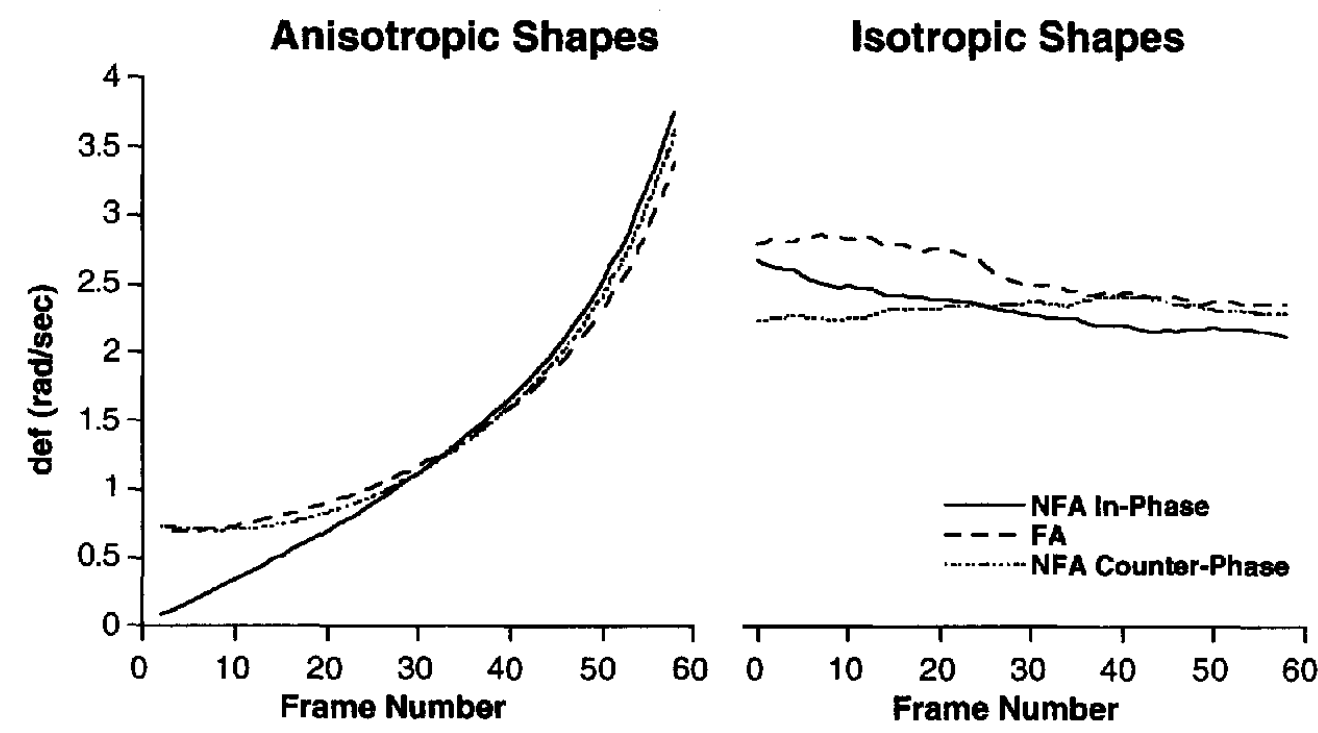

Figure 10. def (deformation) as a function of frame transition in Experiment 4 for one anisotropic shape (left panel) and one isotropic shape (right panel) in the NFA in-phase, FA, and NFA counterphase conditions. NFA $=$ nonfixed-axis rotations; $F A=$ fixed-axis rotations. 
be shown in only one third of the trials. No feedback was given until after the experiment was completed. Otherwise, the procedure was the same as in Experiment 1.

\section{Results and Discussion}

We analyzed the data by using a signal-detection paradigm with the fixed-axis rotation serving as the signal trials and the nonfixed-axis rotation serving as the noise trials. We calculated a $d^{\prime}$ score for each observer by using the percentage of fixed-axis responses on fixed-axis rotation as the hit rate and the percentage of fixed-axis responses on nonfixed-axis rotation as the false-alarm rate. Each $d^{\prime}$ was based on 240 trials, one third of which were signal trials. We calculated the significance of $d^{\prime}$ by using Marascuilo's one-signal significance test (Marascuilo, 1970). The results of this analysis are reported in Table 3.

For isotropic shapes we expected an accurate discrimination between fixed-axis and nonfixed-axis rotations because, in this case, the first-order properties of the optic flow are sufficient for performing the task veridically (see Equation $3)$. For fixed-axis rotations, in fact, both def and $\rho$ remain constant (see Figures 10 and 11) and, therefore, also $f_{\text {def }}$ (def) and $f_{\mathrm{p}}(\rho)$. In these circumstances, the magnitudes of $\sigma_{\text {axis }}^{\prime}$ computed according to Equation 3 take on a constant value in each frame transition of the stimulus sequence. For nonfixed-axis rotations, def is constant and $\rho$ varies. Therefore, the magnitudes of $\sigma_{\text {axis }}^{\prime}$ took on different values in each frame transition of the stimulus sequence. The expected and observed hits, false alarms, misses, and correct rejections rates are reported in Table 4. Consistent with our predictions, all eight $d^{\prime}$ 's were significantly different from zero (see Table 3 ). The average $d$ ' was equal to 1.55 .

The veridical performance obtained with the isotropic shapes is not informative about the underlying perceptual strategy used to recover $\sigma_{\text {axis }}^{\prime}$ because it could be due either to a mathematically correct analysis of the second-order optic flow or to a heuristic analysis of the first-order optic flow. Nevertheless, these results indicate that observers can veridically discriminate between fixed-axis and nonfixed-axis rotations within the stimulus parameters used in Experiment 3.

On the other hand, performance with the anisotropic shapes is informative about the underlying perceptual strategy used to recover $\sigma_{\text {axis }}^{\prime}$ because in this case, a veridical analysis of the second-order optic flow and the heuristic analysis described by Equation 3 make contrasting predictions. In fact, according to Equation 3, we expected that observers would perform veridically in the NFA counterphase condition but would not perform veridically in the FA and NFA in-phase conditions. In the NFA counter-phase
Table 4

Observed and Expected Hits, False Alarms, Misses, and Correct Rejections Rates for Experiment 4

\begin{tabular}{ccccc}
\hline & \multicolumn{4}{c}{ Response } \\
\cline { 2 - 3 } Signal & Observed & Expected & Observed & Expected \\
\cline { 2 - 5 } Yes & Isotropic shapes \\
Yes & .231 & .333 & .115 & 0 \\
No & .102 & 0 & .552 & 667 \\
\hline \multicolumn{5}{c}{ Anisotropic shapes } \\
Yes & .088 & 0 & .35 & .333 \\
No & .245 & .333 & .317 & .333 \\
\hline
\end{tabular}

condition, the magnitudes of def and $\rho$ varied in opposite directions in each frame transition of the stimulus sequence (one increased and the other decreased; see Figures 10 and 11). $\sigma_{\text {axis }}^{\prime}$ derived by Equation 3, therefore, took on different values in each frame transition of the stimulus sequence. Consistently, in $85.8 \%$ of the cases observers veridically judged these displays as nonfixed-axis rotations. In the FA condition, $\rho$ was constant but def varied in each frame transition of the stimulus sequence (see Figures 10 and 11). $\sigma_{\text {axis }}^{\prime}$ derived by Equation 3, therefore, took on different values in each frame transition of the stimulus sequence. Consistently, in $74 \%$ of the cases, observers mistakenly judged these displays as nonfixed-axis rotations. Finally, in the NFA in-phase condition, the magnitudes of def and $\rho$ varied in the same direction in each frame transition of the stimulus sequence. Because $f_{\text {def }}$ and $f_{\rho}$ increased or decreased in phase, the rate of change of their ratio during rotation is smaller than in the counter-phase condition. Therefore, we expected a greater likelihood of fixed-axis responses. Consistent in $91 \%$ of the cases observers mistakenly judged these displays as fixed-axis rotations. The percentages of fixedaxis responses for FA, NFA in-phase, and NFA counterphase conditions are reported in Figure 12.

We predicted that for the anisotropic shapes, observers would produce negative $d^{\prime}$ scores because FA rotations were expected to be judged as NFA rotations (thus, ideally approaching a hit rate of 0 ) and NFA in-phase rotations were expected to be judged as FA rotations (thus, ideally approaching a false alarm rate of 0.33 ; see Table 4). Consistent with this prediction, for the anisotropic shapes all $d^{\prime}$ 's significantly different from zero were negative (see Table 3 ). The average $d^{\prime}$ was equal to -.747 .

In conclusion, the results of Experiment 4 were consistent

Table 3

d'Scores for Experiment 4

\begin{tabular}{lrlrrrrrr}
\hline \multicolumn{1}{c}{ Shape } & \multicolumn{1}{c}{$d^{\prime}$} \\
\hline Isotropic & $1.609^{*}$ & $1.074^{*}$ & $1.377^{*}$ & $1.540^{*}$ & $2.271^{*}$ & $1.866^{*}$ & $1.175^{*}$ & $1.491^{*}$ \\
Anisotropic & $-1.213^{*}$ & -.094 & $-.944^{*}$ & $-1.377^{*}$ & $-.809^{*}$ & -.180 & $-.599^{*}$ & $-.761^{*}$ \\
\hline${ }^{*} p<.01$. & & & & & & & &
\end{tabular}




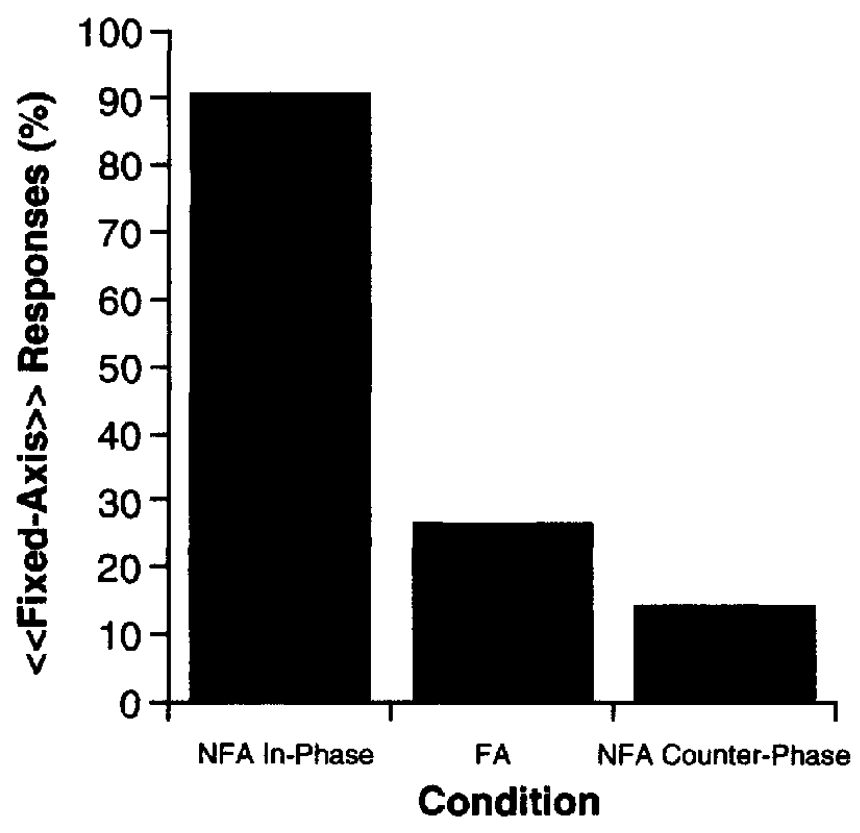

Figure 12. Percentages of fixed-axis judgments in Experiment 4 for rotations NFA in-phase, and NFA counterphase rotations. $\mathrm{NFA}=$ nonfixed-axis rotations; $F A=$ fixed-axis rotations.

with the heuristic analysis described by Equation 3: Observers were able to veridically discriminate fixed-axis from nonfixed-axis rotations for isotropic but not for anisotropic shapes.

\section{General Discussion}

In four experiments, we studied the influence of the first-order properties of the velocity field on the orientation of the axis of rotation perceived in SFM displays. In the first and third experiment, we measured the perceived slant of the axis of rotation; in the second experiment, we measured the perceived tilt of the axis of rotation; and in a fourth experiment, we investigated the discrimination between fixed-axis and nonfixed-axis rotations.

In Experiment 1, we found that observers provided consistent judgments for the slant of the axis of rotation in two-view displays, even if these displays did not provide sufficient information for uniquely recovering the simulated slant of the axis of rotation. The magnitudes of the perceived slant of the axis of rotation were predicted with high accuracy by the arc tangent of the ratio of def and $\rho$.

In Experiment 2, we found that the tilt of the axis of rotation was perceived in two-view apparent motion sequences with a very small error. This result shows that the first-order properties of the velocity field are perceptually effective for recovering this parameter of the projected motion.

In Experiment 3, we found that the slant of the axis of rotation was systematically misperceived in multiview displays that provided information theoretically sufficient for a veridical recovery of this aspect of the projected motion.
These misperceptions closely resembled the biases found in Experiment 1 . The inaccuracy of performance for multiview displays is also consistent with what Norman and Todd (1994) reported. They used multiview displays and obtained discrimination thresholds for how well observers could discriminate whether two shapes rotated about either the same axis or about two different axes with different slants. When the two objects were spatially separated (did not intersect in the projected image), Norman and Todd (1994) found relatively large errors (high discrimination thresholds for detecting the differing slants of the axes of rotation). These difference thresholds were about $20^{\circ}$ when the surfaces had visible texture elements and went up to $50^{\circ}$ when only profiles were visible.

In Experiment 4, we found that the discrimination between fixed-axis and nonfixed-axis rotations can be systematically biased by manipulating the properties of the firstorder flow. Consistent with the heuristic analysis described by Equation 3, we created stimulus displays that allowed veridical performance (isotropic shapes) and stimulus displays in which performance was highly inaccurate (anisotropic shapes).

The systematic misperceptions of the axis of rotation revealed by the present investigation provide converging evidence for the view that human perception of SFM may be restricted to an analysis of the first-order optic flow. Evidence showing that perceived 3-D structure and motion in SFM displays is based primarily on two views, even if more views are available, has been provided by numerous investigators (e.g., Norman \& Todd, 1993; Todd et al., 1988; Todd \& Bressan, 1990; Todd \& Norman, 1991). Even though some reports indicate that second-order information may be partially available to human observers (Domini, Caudek, Turner, \& Favretto, in press; Norman \& Todd, 1993; Perotti, Todd, \& Norman, 1996), many SFM phenomena can be accounted for by a heuristic analysis of the first-order optic flow. The heuristic approach to SFM was first proposed by Braunstein $(1972,1994)$, and this view is currently shared by several investigators. In various studies, for example, it has been pointed out that the magnitudes of depth and slant perceived in SFM could be derived according to a simple relative-motion heuristic process after removing the curl component of the optic flow (e.g., Caudek \& Proffitt, 1993; Durgin et al., 1995; Liter et al., 1993; Proffitt et al., 1992). A number of studies have also directly contrasted the properties of the first-order velocity field with the structural and dynamic properties of the projected objects. These investigations have revealed that a heuristic analysis of the first-order flow may be responsible for many misperceptions of human SFM.

Domini et al. (1994) and Domini et al. (1997) investigated the perception of rigidity. They found that displays with a low variability of the deformations of the individual triplets of image features tended to be judged rigid more often than those with a high variability of the deformations, regardless of whether they simulated a rigid transformation or not. Caudek et al. (1994) and Domini, Caudek, and Richman (in press) investigated the perception of depth-order relations. They found that by manipulating the first-order optic flow, it 
was possible to bias the perceptual signing of the near-far relations so as to reveal global inconsistencies in the perceived ordinal structure. For probes placed on a closed surface, in fact, the integration of the local depth-order judgements was found to produce a paradoxical violation of the transitivity principle (A > B, B > C, C > A) inconsistent with the properties of either Euclidean or affine 3-D structures. Domini and Braunstein (in press) investigated the perception of depth magnitudes. They found that the perceived depth separation of two probe points was influenced by the def produced by the surface (or the surfaces) on which the probes were located. These results are consistent with a heuristic analysis that derives the slant of a planar surface as a nonlinear function of def. This heuristic model predicts distortions in the perceived depth separation of a pair of points located on a generic curved surface such that the integration of the depth judgments on closed paths, in general, does not vanish. Empirical results confirmed this prediction and suggested that judged depth from moving orthogonal projections cannot be represented in either a Euclidean or an affine space. Domini et al. (in press) investigated the discrimination between constant and variable 3-D angular velocities. They found that displays with a low variability of the defs of the individual triplets of image features tended to be judged as rotating with a 3-D constant angular velocity more often than those with a high variability of the defs, regardless of whether they simulated a 3-D constant angular velocity or not. Domini et al. (1995) and Domini and Caudek investigated the perception of slant. They found that when the def and the simulated slant were pitted one against the other, perceived slant depended on the def rather than on the simulated slant.

The evidence provided by these studies, therefore, indicates that many aspects of human perception of SFM can be accounted for by a heuristic analysis of the first-order optic flow. The formulation of more precise quantitative models relating the properties of the first-order optic flow to the structural and dynamic properties perceived in SFM and to the understanding of the mutual relations among the heuristics accounting for different aspects of perceptual performance remains a question for future research.

\section{Conclusion}

For two-view and multiview displays, the perceived slant of the axis of rotation of planar surfaces and spherical volumes is well-predicted by the ratio between the def and the component of rotation about an axis orthogonal to the image plane. If this ratio is kept constant in each frame transition of the stimulus sequence (or it is varied), then the stimuli tend to be judged as fixed-axis rotations (or as nonfixed-axis rotations), regardless of whether they simulate a fixed-axis rotation or not.

\section{References}

Braunstein, M. L. (1962). The perception of depth through motion. Psychological Bulletin, 59, 422-433.

Braunstein, M. L. (1972). Perception of rotation in depth: A process model. Psychological Review, 79, 510-524.
Braunstein, M. L. (1994). Decoding principles, heuristics and inference in visual perception. In G. Jansson, S. S. Bergstrom, \& W. Epstein (Eds.), Perceiving events and objects (pp. 436-446). Hillsdale, NJ: Erlbaum.

Braunstein, M. L., \& Andersen, G. (1981). Velocity gradients and relative depth perception. Perception \& Psychophysics, 29, 145-155.

Braunstein, M. L., \& Andersen, G. (1984). A counterexample to the rigidity assumption in the visual perception of structure from motion. Perception, 13, 213-217.

Braunstein, M. L., Hoffman, D. D., \& Pollick, F. E. (1990). Discriminating rigid from nonrigid motion: Minimum points and views. Perception \& Psychophysics, 47, $205-214$.

Braunstein, M. L., \& Tittle, J. S. (1988). The observer-relative velocity field as the basis for effective motion parallax. Journal of Experimental Psychology: Human Perception and Performance, 14, 582-590.

Caudek, C., Domini, F., \& Gerbino, W. (1994, November). Paradoxical perception of depth-order relations in SFM displays. Poster presented at the 35th Annual Meeting of the Psychonomic Society, St. Louis, MO.

Caudek, C., \& Proffitt, D. R. (1993). Depth perception in motion parallax and stereokinesis. Journal of Experimental Psychology: Human Perception and Performance, 19, 32-47.

Domini, F., \& Braunstein, M. L. (in press). 3D structure from motion is neither Euclidean nor affine. Journal of Experimental Psychology: Human Perception and Performance.

Domini, F., \& Caudek, C. (in press) Perceiving surface slant from deformation of optic flow. Journal of Experimental Psychology: Human Perception and Performance.

Domini, F., Caudek, C., \& Gerbino, W. (1995). Perception of surface attitude in structure from motion displays [Abstract]. Investigative Ophthalmology \& Visual Science, 36, 1694.

Domini, F., Caudek, C., \& Proffitt, D. (1994). Discriminating rigid from nonrigid motion in the kinetic depth effect. Perception, 23 (Suppl.), 56a.

Domini, F., Caudek, C., \& Proffitt, D. R. (1997). Misperceptions of angular velocities influence the perception of rigidity in the kinetic depth effect. Joumal of Experimental Psychology: $\mathrm{Hu}$ man Perception and Performance, 23, 1111-1129.

Domini, F., Caudek, C., \& Richman S. (in press). Distortions of depth-order relations and parallelism in structure from motion. Perception \& Psychophysics.

Domini, F., Caudek, C., Turner, \& Favretto, A. (in press). Discriminating constant from variable angular velocities in the kinetic depth effect. Perception \& Psychophysics.

Durgin, F. H., Proffitt, D. R., Olson, T. J., \& Reinke, K. S. (1995). Comparing depth from motion with depth from binocular disparity. Joumal of Experimental Psychology: Human Perception and Performance, 21, 679-699.

Eby, D. W. (1992). The spatial and temporal characteristics of perceiving 3-D structure from motion. Perception \& Psychophysics, 51, 163-178.

Hildreth, E. C., Grzywacz, N. M., Adelson, E. H., \& Inida, V. K. (1990). The perceptual buildup of three-dimensional structure from motion. Perception \& Psychophysics, 48, 19-36.

Hoffman, D. D. (1982). Inferring local surface orientation from motion fields. Journal of the Optical Society of America, A72, 888-892.

Kaiser, M. K. (1990). Angular velocity discrimination. Perception \& Psychophysics, 47, 149-156.

Kaiser, M. K., \& Calderone, J. B. (1991). Factors influencing perceived angular velocity. Perception \& Psychophysics, 50, $428-434$

Koenderink, J. J. (1986). Optic flow. Vision Research, 26, 161-180. 
Koenderink, J. J., \& van Doorn, A. J. (1975). Invariant properties of the motion parallax field due to the movement of rigid bodies relative to an observer. Optica Acta, 22, 773-791.

Koenderink, J. J., \& van Doorn, A. J. (1976). Local structure of movement parallax of the plane. Journal of the Optical Society of America, A 66, 717-723.

Lappin, J. S., Doner, J. F., \& Kottas, B. L. (1980). Minimal conditions for the visual detection of structure and motion in three dimensions. Science, 209, 717-719.

Liter, C. J., Braunstein, M. L., \& Hoffman, D. D. (1993). Inferring structure from motion in two-view and multiview displays. Perception, 22, 1441-1465.

Loomis, J. C., \& Eby, D. W. (1988). Perceiving structure from motion: Failure of shape constancy. In Proceedings of the Second International Conference of Computer Vision (pp. 383391). Washington, DC: IEEE Computer Society Press.

Loomis, J. C., \& Eby, D. W. (1989). Relative motion parallax and the perception of structure from motion. In Proceedings of the IEEE Workshop on Visual Motion (pp. 204-211). Washington, DC: IEEE Computer Society Press.

Marascuilo, L. A. (1970). Extensions of the significance test for one-parameter signal detection hypotheses. Psychometrika, 35, 237-243.

McKee, S. P. (1981). A local mechanism for differential velocity detection. Vision Research, 21, 491-500.

Norman, J. F., \& Todd, J. T. (1993). The perceptual analysis of structure from motion for rotary objects undergoing affine stretching transformations. Perception \& Psychophysics, 53, 279-291.

Norman, J. F., \& Todd, J. T. (1994). Perception of rigid motion in depth from the optical deformations of shadows and occlusion boundaries. Journal of Experimental Psychology: Human Perception and Performance, 20, 343-356.

Perotti, V. J., Todd, J. T., \& Norman, J. F. (1996). The visual perception of rigid motion from constant flow fields. Perception \& Psychophysics, 58, 666-679.
Pollick, F. E. (1994). Perceiving shape from profiles. Perception \& Psychophysics, 55, 152-161.

Pollick, F. E. (1997). The perception of motion and structure in structure-from-motion: Comparison of affine and Euclidean formulations. Vision Research. 37(3), 347-353.

Pollick, F. E., Nishida, S., Koike, Y., \& Kawato, M. (1994). Perceived motion in structure from motion: Pointing responses to the axis of rotation. Perception \& Psychophysics, 56, 91-109.

Proffitt, D. R., Rock, I., Hecht, H., \& Schubert, J. (1992). Stereokinetic effect and its relation to the kinetic depth effect. Journal of Experimental Psychology: Human Perception and Performance, 18, 3-21.

Sperling, G., Landy, M. S., Dosher, B. A., \& Perkins, M. E. (1989). Kinetic depth effect and identification of shape. Journal of Experimental Psychology: Human Perception and Performance, IS, 826-840.

Todd, J. T., Akerstrom, R. A., Reichel, F. D., \& Haynes, W. (1988). Apparent rotation in three-dimensional space: Effects of temporal, spatial, and structural factors. Perception \& Psychophysics, $43,179-188$.

Todd, J. T., \& Bressan, P. (1990). The perception of 3-dimensional affine structure from minimal apparent motion sequences. Perception \& Psychophysics, 48, 419-430.

Todd, J. T., \& Norman, J. F. (1991). The visual perception of smoothly curved surfaces from minimal apparent motion sequences. Perception \& Psychophysics, 50, 509-523.

Wallach, H., \& O'Connell, D. N. (1953). The kinetic depth effect. Journal of Experimental Psychology, 45, 205-217.

Received June 17, 1996

Revision received October 17, 1996 Accepted January 31, 1997 